\title{
UN CONTRATO INÉDITO DE JUAN DE JUANES. EL RETABLO DE LA COFRADÍA DE LA SANGRE DE CRISTO DE VALENCIA (1539)
}

\author{
Mercedes Gómez-Ferrer y Juan Corbalán de Celis \\ Universitat de València
}

\begin{abstract}
Se presenta un contrato inédito suscrito por la Cofradía de la Sangre de Cristo de la ciudad de Valencia con Vicente y Juan Macip en 1539. En él se especifica que sea pintado por Juan, más conocido con posterioridad como Juan de Juanes. Se refuerza la hipótesis que permite concretar a Juan Macip como el pintor más importante del taller de los Macip durante la década de los 30, al tiempo que se llena un vacío documental entre la entrega del retablo del gremio de plateros (1534-1539) y la contratación del retablo de Fuente la Higuera (1548). Se constata igualmente la popularidad de la iconografía de los siete derramamientos de la Sangre de Cristo y la procesión de los disciplinantes.
\end{abstract}

Palabras clave: Pintura renacentista; Retablos; Juan de Juanes; Vicente Macip; Cofradía de la Sangre; Valencia.

\section{JUAN DE JUANES: AN UNPUBLISHED CONTRACT FOR THE ALTARPIECE OF THE BROTHERHOOD OF THE BLOOD OF CHRIST, VALENCIA (1539)}

This article is based on an unpublished contract signed in 1539 by Vicente and Juan Macip for an altarpiece for the Valencian Brotherhood of the Blood of Christ. The agreement stipulates that Juan, later known as Juan de Juanes, had to paint the scenes. Thus the document reinforces the hypothesis that Juan Macip was the most important painter in the Macip workshop during the 1530 's. It also fills a documental void in between the completion of the Silversmith's Guild altarpiece (1534-1539) and the contract for the Fuente la Higuera altarpiece (1548). This study also verifies the popularity of the iconography of the seven sheddings of the Blood of Christ and processions of disciplinants.

Key words: Renaissance painting; Altarpiece; Juan de Juanes; Vicente Macip; Blood of Christ Brotherhood; Valencia.

La obra de Juan de Juanes sigue suscitando muchísimo interés entre los estudiosos dedicados a la pintura renacentista. En los últimos años se ha producido un fascinante debate sobre la personalidad de este pintor y la de su padre, Vicente Macip. Muchos historiadores han intervenido aceptando o cuestionando algunas de las propuestas que se han vertido y que han sido ciertamen- 


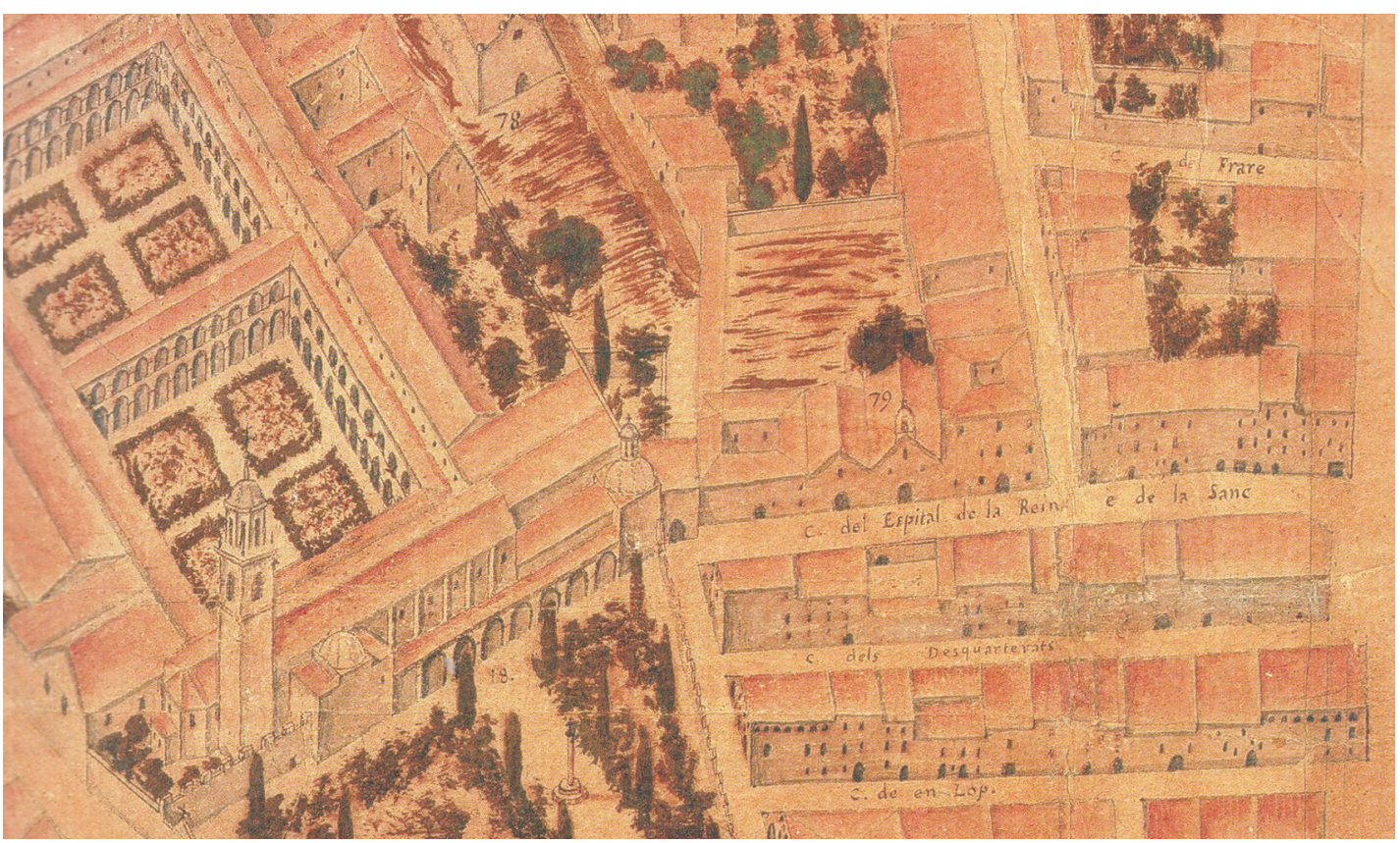

Fig. 1. Detalle del plano de la ciudad de Valencia de Tosca (1704), con el n. ${ }^{\circ}$ 79, casa de la cofradía de la Sangre.

te polémicas ${ }^{1}$. Por un lado, parece comúnmente aceptado que la desconocida pintura de Vicente Macip, con una cronología que se adelantaba al primer cuarto del siglo XVI puede identificarse con la que se consideraba del anónimo Maestro de Cabanyes ${ }^{2}$. Macip se documentaba con claridad solo a partir de 1529, año en que comienza a pintar el retablo de Segorbe. Sin embargo, su nacimiento hacia 1468 o 1470, avanzaba muchísimo la cronología de sus obras, pues había más de treinta años de su pintura que no se habían identificado. Esta propuesta de considerar como obras de Vicente Macip las del anónimo maestro de Cabanyes, resultaba convincente. Lo que ya no lo era tanto, fue el argumento del cambio radical en su modo de pintar a raíz de la presencia en la ciudad de Valencia de la obra de Sebastiano del Piombo, que llegaba a la ciudad con el embajador Vich en 1521. El conocimiento de estas obras aparentemente supuso una conversión y un cambio sustancial en su pintura, con lo que a Macip se le atribuyeron algunas de las obras que durante muchos años habían formado parte del catálogo de Juan de Juanes.

Casi todas las situadas entre los años 30 y 40. Juanes quedaba un tanto relegado tras esta propuesta y casi y únicamente adquiría personalidad propia tras la muerte de su padre en 1551. Esta última hipótesis es la que se ha cuestionado más y se ha reformulado últimamente, porque

1 A la bibliografía tradicional sobre Vicente Macip y Juan de Juanes básicamente recogida en el libro de Albi, 1979, se han ido sumando en los últimos años una serie de textos que incidían de distinta forma en la personalidad de ambos pintores. Tras unos primeros artículos que se detallan en la siguiente nota, Fernando Benito propuso una identificación de Vicente Macip con el Maestro de Cabanyes y un cambio en el catálogo pictórico de Vicente Macip, que se ampliaba enormemente cogiendo buena parte de la obra hasta entonces considerada como de Juan de Juanes. Un resumen de estas propuestas se realizaron en el Catálogo de la Exposición, Benito, 1997. La reconsideración de esta propuesta por parte de varios autores y especialmente Company, X y Tolosa, L, 1999a: 263-278, motivó un cambio y una vuelta a un catálogo más amplio de Juan de Juanes, con un resumen de lo acontecido y de la bibliografía aparecida entre una y otra exposición en Benito, 2000.

${ }^{2}$ Benito, 1993: 223-244. Corroborada con otras investigaciones como la de Samper, 1994: 35-38. 
resulta muy poco creíble que un pintor con unos modos tan arraigados y propios de una tradición cuatrocentista y con una edad avanzada, ante la visión de unas obras, cambie radicalmente su modo de $\operatorname{pintar}^{3}$. A Juanes, por tanto, se le han devuelto estas obras que tras esta propuesta habían pasado a manos de su padre y su catálogo ha sido restituido. Es más, no solo se le han devuelto algunas de estas obras, sino que incluso se plantea la posibilidad de que su actividad también sea mucho más precoz y que parte del cambio que ya se observaba en el retablo de Segorbe se pueda deber precisamente a su mano. A toda esta disquisición se han sumado datos documentales sobre diversos retablos de difícil adscripción, corroboración de obras acertadamente atribuidas, depuración de la biografía personal y familiar ${ }^{4}$, reconsideración historiográfica sobre la fortuna de su pintura ${ }^{5}$ o clarividentes observaciones sobre el contexto social ${ }^{6}$ en el que se movió, que vienen a incidir en la interesante personalidad de uno de los pintores más afamados en el medio valenciano del siglo XVI.

No obstante, y a pesar de todos estos logros, siguen planteándose dudas e incertidumbres. Los campos de investigación siguen abiertos porque cuestiones tan importantes como el aprendizaje de Juan de Juanes, su posible viaje a Italia, la delimitación de su actividad pictórica en los años iniciales en los que despunta o la composición del taller de los Macip son algunos de los asuntos que aún hoy en día nos interrogan. Por ello, los hallazgos documentales son también bienvenidos en la medida que pueden dar luz a estos estudios y más si se refieren al difícil periodo en el que padre e hijo comparten taller. Estos años especialmente controvertidos se sitúan entre 1531, en que se menciona el primer pago a Juan Macip por estrenas para el retablo mayor de la catedral de Segorbe, y 1551, fecha en que muere su padre Vicente Macip. Se trata por tanto de 20 años de convivencia entre dos pintores con una fuerte personalidad y en donde cada vez más claramente se observa la prevalencia del hijo, del pintor más joven, frente al padre o cabeza de taller que, no obstante, es en muchas ocasiones el que firma los contratos. Lo cierto es que las recientes aportaciones documentales vienen a incidir en la práctica aseveración de que buena parte de lo pintado en la década de los años 30 es de la mano de Juan Macip, conocido con posterioridad como Juan de Juanes. Y esto incide en el creciente protagonismo de Juanes como pintor destacado con una gran madurez, a pesar de su juventud, desde fechas mucho más tempranas a las que hasta no hace mucho se venían considerando.

Los datos que presentamos en este artículo corresponden a un retablo del cual no existía noticia alguna hasta el momento. Por situarse precisamente en los años en los que se está cuestionando la actividad de Juanes y en los que su padre está aún presente en el taller, creemos que puede resultar de interés para volver a incidir en que la línea de trabajo que se ha abierto sobre Juan de Juanes es la correcta y que es sobre la que se debe continuar trabajando. Se trata de un retablo que encargaron los cofrades de la Cofradía de la Preciosísima Sangre de Cristo de Valencia en 1539 al taller de los Macip. El contrato se suscribió entre los representantes de la cofradía y los pintores Vicent Macip y Joan Macip, padre e hijo, el 29 de diciembre de 15397, con unas cláusulas muy concretas que apuntalan lo anteriormente mencionado. A continuación detallaremos la historia de la cofradía para enmarcar el retablo en el proceso de fundación de una nueva institución en la Valencia del siglo XVI y con posterioridad analizaremos los datos que podemos

\footnotetext{
3 Además del artículo citado en la primera nota, insistían en lo poco convincente de esta propuesta Company, y Tolosa, 1999b: 50-61, indicando que la intervención de Juanes en el retablo de Segorbe también pudo ser decisiva.

${ }^{4}$ Samper, 2001: 163-171, y Hernández, y Company, 2006: 211-269, donde se realiza también una importante reconsideración de todo el debate sobre Macip y Juanes, resumiendo la situación y el estado de la cuestión actual, y añadiendo la bibliografía aparecida hasta la fecha sobre este tema.

${ }^{5}$ Falomir, 1999: 123-147. También resultó de gran interés la publicación de Bassegoda, 1995: 165-172.

6 Falomir, 2006: 271-288.

7 Archivo de Protocolos Patriarca de Valencia (APPV), Protocolo 16049, notario: Pere Mir, ver apéndice documental, documento n. ${ }^{\circ} 1$.
} 


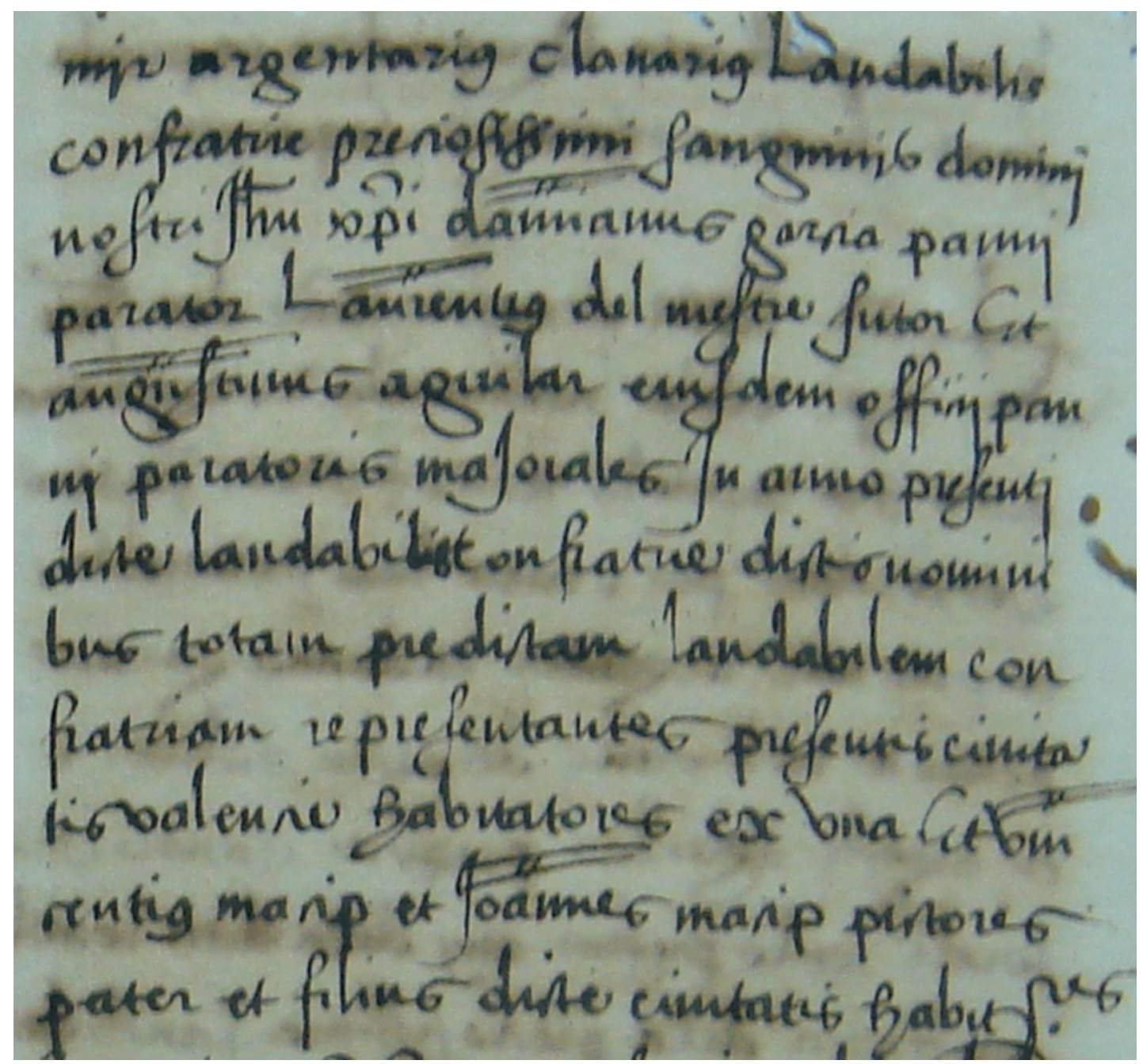

Fig. 2. Detalle del contrato entre los miembros de la cofradía de la Sangre y Vicente Macip y Juan Macip, padre e hijo, pintores.

extraer a partir de la documentación sobre el contrato de pintura con los Macip y de escultura con Baltasar Fortuny ${ }^{8}$ y Martín Sancho ${ }^{9}$.

\section{La Cofradía de la Sangre}

La devoción a la Sangre de Cristo, que se remonta a la antigüedad cristiana, comienza a popularizarse durante la Edad Media con su incorporación a las cofradías de disciplinantes que aparecen en la península ibérica a finales del siglo XV, como continuadoras de las fundadas,

8 APPV, protocolo: 16048, notario Pere Mir, ver apéndice documental, documento n. ${ }^{\circ} 2$. 
primero en Brujas y después en Mantua, a mediados de dicho siglo. En los primeros años de la centuria siguiente comienzan a surgir en los territorios del antiguo reino de Aragón cofradías bajo el título de la Preciosísima Sangre de Cristo, similares a las de la Vera Cruz aparecidas simultáneamente en Castilla ${ }^{10}$.

En Valencia el culto a la Sangre de Cristo que, con las hermandades de penitentes y disciplinantes participantes en la Semana Santa, venía de antiguo ${ }^{11}$, toma gran auge en el primer tercio del siglo XVI ${ }^{12}$, influyendo quizá en ello la publicación por esas fechas de un pequeño poema compuesto por el notario Andrés Martín Pineda ${ }^{13}$, que exaltaba las excelencias de la Sangre de Cristo y mostraba, mediante unos grabados, los siete momentos en que, según la tradición recogida por el dominico Cavalca ${ }^{14}$, nuestro Señor derramaba su preciosísima sangre ${ }^{15}$.

El 15 de marzo de 1535, el vicario general aprobaba las Constituciones de la recién erigida Confraría de la Santissima Sanch de Jesu Christ ${ }^{16}$, que un grupo de gente de la ciudad vinculada a profesiones artesanales y del comercio había fundado poco tiempo antes en la iglesia de San Miguel y San Dionisio, en la capilla allí existente bajo invocación de la Sangre, con la anuencia del arzobispo y del señor duque de Calabria ${ }^{17}$. Estos primeros cofrades, pertenecientes en su totalidad a los distintos gremios de la ciudad de Valencia, desbordados desde el primer momento por el gran número de devotos que acudieron a formar parte de esta cofradía, pronto deciden buscar un solar donde poder construir una casa amplia y suficiente para poder reunirse, edificar una capilla para sus devociones y hacer penitencia los disciplinantes la víspera de Jueves Santo.

En la junta que tiene lugar unas semanas después, el jueves día 6 de mayo, en la calle de Quart, en la casa gremial de los peraires, que éstos le cedieron para la ocasión ${ }^{18}$, deciden comprar en el antiguo cementerio de los moros, junto a la iglesia de San Miguel, que ya se encontraba en desuso ${ }^{19}$, terrenos que entonces pertenecían a esta iglesia ${ }^{20}$. Al año siguiente, el 16 de julio de 1536, como la cofradía se encontraba con pocos medios y no tenía dinero suficiente para poder edificar, se cargan un censal para poder hacer frente a los gastos derivados de todo ello. Unos días después, el 28, permutaban con el notario Joan Guimerá, dueño del llamado

10 Labarga, 1999: 381-392.

11 Cruilles, 1876: 436-439.

12 Martínez Romero, 2001.

${ }^{13}$ Martín Pineda, 1521, un pequeño libro de 4 páginas cuyos grabados recogen los siete derramamientos de la sangre de Cristo.

${ }_{14}$ Navarro Espinach, 2006: 583-611, cita el texto del dominico Cavalca (1270-1342) difundido en el siglo XV, Mirall de la Creu, versió catalana del segle XV per Pere Busquets, ed. de A. Gallina, Barcelona, 1967.

15 Según Domenico Cavalca, las siete veces que nuestro Señor derramó su sangre por nosotros durante su vida fueron: 1) en la circuncisión; 2) sudando gotas de sangre en el huerto de los Olivos; 3) cuando fue flagelado y azotado en la columna; 4) coronado de espinas; 5) cuando le clavaron las manos en la cruz; 6) cuando le clavaron los pies; y 7) cuando Longinos le clavó la lanza en el costado.

16 Teixidor: 1895.

17 Archivo de Protocolos Patriarca de Valencia (APPV), Protocolo 16049, notario: Pere Mir.

18 En esa junta figuran un total de 177 cofrades, que no eran todos los que formaban la cofradía, y que aparecen reseñados con más de sesenta oficios diferentes.

19 APPV. Protocolo 16013, notario Onofre Çapena.

${ }^{20}$ En agosto de 1539, después de haber comprado un viejo edificio, y no necesitando ya el solar del antiguo cementerio, deciden sacarlo a subasta, vendiéndolo por 40 libras al cofrade Joan Mas, que decía "lo hacía por beneficio de la cofradía, y no porque tuviese necesidad de aquel", pero al cabo del tiempo y viendo que no se hacía efectiva la dita, le reprochan que hubiese hecho una puja simulada, pero en consideración de que la cantidad ofrecida era mucho más de lo que valía el solar, y de que lo había hecho sin mala intención, deciden darla por nula. APPV. Protocolo 16048, notario Pere Mir. 
horno de la morería, que se encontraba frente al solar que había sido cementerio, un corral que había junto al horno ${ }^{21}$. Pero viendo, quizá, los excesivos gastos que tendrían que acometer para edificar de nueva planta una casa para la cofradía que resultase amplia y suficiente para cubrir las necesidades que tenían de sala para juntas, capilla con enterramiento, de un lugar espacioso para reunirse los cofrades y vestirse para la procesión de la noche del Jueves Santo, así como para reponerse y curarse de las llagas y heridas que se producían en ese día, deciden buscar algún edificio que, con pocas obras, fuese capaz de dar satisfacción a todas estas necesidades de espacio ${ }^{22}$.

Eligieron para ello el antiguo Hospital de la Reina, que había dejado de funcionar como tal en 1512 cuando, junto con otros de Valencia, fue incorporado al hospital de Inocentes, que pasaría desde entonces a denominarse Hospital General. Este establecimiento, perteneciente a la parroquia de san Martín, se encontraba situado junto a los muros del monasterio de san Francisco, ocupando casi una manzana completa. Estaba compuesto por dos naves, diversas porchadas y dependencias, y la parte llamada lo statge del verguer, teniendo la mayor de las naves fachada a la calle llamada del Hospital de la Reina, la otra, la navada chica, estaba dentro del huerto que había a espaldas de la anterior, con entrada por un callejón que venía desde la calle dels reglons, junto a la puerta de la capilla de los genoveses, acequia de Rovella en medio. El edificio, que había sido comprado por particulares, pertenecía al menos desde 1530 a Joan Bautista Riço, mercader, que lo tenía arrendado por partes, habiéndose instalado en el antiguo huerto, ya transformado en corral, un hostal y parador de carros, que se llamaría lo hostal e carretería de la Sanch y daría nombre al callejón por el que se accedía al mismo ${ }^{23}$. Formalizaron la compra el 11 de marzo de 1538, siendo el precio estipulado de 27.333 sueldos que pagarían, parte en metálico en ese momento y parte mediante unos censales de los que deberían responder anualmente en dos pagas.

Poco después, en julio de 1539, y con el fin de atraer a los fieles a la nueva capilla que edificaban o adaptaban, y obtener los disciplinantes de la cofradía las indulgencias que otras cofradías ya disfrutaban, acordaron suplicar al sumo Pontífice que les concediese algunas gracias e indulgencias, así como alguna bula o jubileo, para lo cual acordaron enviar a Roma 70 ducados de oro, para hacer frente a los gastos de la tramitación.

Los primeros tiempos de andadura de la nueva cofradía fueron dificultosos y costosos. A los gastos que le supuso la compra del antiguo Hospital, la obtención de las bulas, el compromiso

21 APPV, Protocolo 16016, notario Onofre Çapena, corral lindante con la casa del entonces clavario Luis Martínez, con el horno, y con dicho cementerio. El notario les cedía un corral que tenía enfrente del horno, donde guardaba la leña, lindante por una parte con la iglesia de San Miguel y de la otra con casa de Antoni Forner.

22 Según Cruilles, una vez comprada la casa posada, huerto y solares que habían sido hospital, levantaron una capilla y sus dependencias bajo la dirección del maestro de obras Bernardo Juan Cetina. La capilla se levantaría o reformaría entre marzo de 1538, fecha en la que realizan la compra, y octubre de 1540, fecha en la que obtienen permiso para poder celebrar oficios en ella.

${ }^{23}$ Hacia 1530 tenía arrendado el edificio Joan Delra, carreter o mestre de axa, el cual había modificado casi todas sus entradas para adaptarlo a su nuevo uso, llegando a demoler el enmarcado de piedra de la puerta principal. Biço lo demandó ante el justicia civil y fue condenado a dar fianzas en el sentido de que una vez acabase el alquiler debería devolver el edificio a su estado original. Un par de años después de la compra del Hospital por la cofradía, como habían tenido pleito con Delra porque éste se negaba a devolver las puertas a su estado original, y estaba a punto de cumplirse el tiempo de su alquiler, no le renuevan el contrato y alquilan la carretería por tiempo de doce años, y seis de respit, empezando a contar desde marzo de 1541, a Jaume Saragoça, por precio de 33 libras anuales. En 1559 venden el hostal o carretería a Pere Pasqual, labrador, y a su yerno Antoni Catalá, fabricantes de arados, por 24.000 sueldos, con la condición de que el dominio y la posesión de las casas carreterías sería de la Cofradía mientras no se liquidase el precio de ellas. APPV. Protocolos 16048, 16049, 16061, notario Pere Mir. Al final la venta no debió llevarse a efecto, pues según Teixidor en 1565 la cofradía vendía estas mismas casas a Jaume Corberan de Let. 
de sufragar los costes del retablo, hay que añadirles los derivados del pleito con el fiscal de la hacienda real sobre el pago de los derechos de amortización ${ }^{24}$. La fundación de la cofradía, con capilla abierta a los cofrades y a los numerosos fieles con los que cuenta en esos momentos la ciudad de Valencia que sentían una especial devoción a la Sangre, suponía un menoscabo para los ingresos de la parroquia de san Martín ${ }^{25}$, a la que vimos pertenecían, y para la iglesia del colindante monasterio de san Francisco ${ }^{26}$, con las que finalmente llegan a acuerdos.

$\mathrm{Su}$ andadura fue más o menos estable hasta que a mediados del siglo XVIII, sobre los terrenos que ocupaba el antiguo parador, el arzobispo Mayoral levantó la casa de Enseñanza. Entonces se utilizó como capilla de la casa hasta que se decidió construir una iglesia al otro lado bajo la advocación de Santa Rosa de Lima. No obstante, la iglesia de la Sangre se renovó en 1766 y la cofradía siguió funcionando. Cuando el Ayuntamiento de Valencia quiso completar todo el solar de su edificio con la parcela recayente a la calle de la Sangre, adquirió de la Archicofradía de la Santísima Sangre de la parroquia de San Agustín el espacio que ocupaba aquella iglesia de la Sangre ${ }^{27}$.

\section{El retablo de la Cofradía de la Sangre}

El proceso de encargo del retablo se enmarca, por tanto, después del verano de 1539 en el momento inicial de fundación de la cofradía, establecidos en el antiguo Hospital de la Reina cuando ya disponen de capilla que deben ornamentar. En primer lugar, el 17 de noviembre de 1539 contrataban con el escultor Baltasar Fortuny la confección de la base de madera sobre la que aquel se pintaría, indicándole que en el centro del mismo debería haber una hornacina en la que se pondría la figura del ecce homo cuando es llevado por los judíos ante Pilatos. Para la confección de esta figura, ese mismo día, contrataban con el imaginero Martín Sancho la talla, en madera de ciprés, de un gran ecce homo que debería presidir el retablo. Al mes siguiente se contrataba con los Macip la pintura del retablo y de la talla.

Por un lado, sabemos que el escultor Baltasar Fortuny tenía que seguir una traza en papel para el retablo que se hallaba en manos del clavario de la cofradía y que describe las medidas que

${ }^{24}$ En el año 1540 se pagan a los distintos abogados que intervienen en el proceso 1.806 sueldos. APPV. Protocolo 16018 , notario Onofre Çapena.

25 En octubre de 1540, los cofrades junto con don Luis de Castellvi, canónigo de la Seo y rector de San Martín, que ya les había dado todas las facilidades de palabra para que pudiesen erigir la cofradía attes a que la obra es pía, y atendiendo también a que en dicha casa ia antigament y celebraven officis divinals y si exercitaven altre obres pies, firmaban ciertas capitulaciones por las que se regulaba la celebración de misas y oficios divinos en la capilla de la cofradía, y por esta oferta que se les hacía deberían pagar anualmente 30 sueldos, y para cualquier celebración deberían contar primero con el clero de la parroquia, al que se le pagaría las cantidades acostumbradas, ver APPV. Protocolo 16049, notario Pere Mir.

26 APPV. Protocolo 16019, notario Pere Mir, Al año siguiente, en el mes de febrero, son los frailes de san Francisco los que protestan ante los jueces apostólicos y ante la gobernación por la apertura de la nueva capilla. Al final llegan a un arreglo amistoso tras el cual acordaron que los cofrades no podrían decir sermones en la casa de la cofradía, durante el tiempo de Cuaresma, los viernes y domingos, ni hacer cantar el salmo del miserere mei en dichos viernes. Además, en las fiestas que se celebran todos los años en honor de la Inmaculada, y del glorioso padre san Francisco, no podrían hacer decir misa cantada, ni sermón, pudiendo celebrar cualquier otra solemnidad y fiestas en otras jornadas en las cuales en la iglesia del monasterio no se hiciese fiesta solemne. Los bacines y colectas que quisiesen tener y hacer tendrían que hacerlo dentro de la Casa, no pudiendo en ninguna forma salir fuera de las puertas para pedir limosna. Les permitían también que pudiesen tener una campana a forma de campanar de hermita sobre el tejado. Los cofrades, en agradecimiento, les entregaron 200 sueldos in caritatem y subvención de los gastos del notario.

27 Corbín, 1988, y Alejos, 1999: 84-87. 
tenía el conjunto. Un retablo de considerables dimensiones, de 24 palmos de alto por 14 de ancho ( $5 \mathrm{~m}$ x $3 \mathrm{~m}$ aprox.), que debía albergar los numerosos compartimentos que luego se detallan en la capitulación. Por este conjunto de madera el escultor cobraría un total de 15 ducados de oro. Por otro lado, se contrata con el imaginero la obra de bulto de la figura del Ecce Homo, una figura también de notables dimensiones, de 7 palmos de altura, casi metro y medio de alta, que debía ajustarse a un modelo de arcilla que tenía en sus manos el clavario. Por esta obra, el imaginero Martín Sancho iba a cobrar un total de 10 ducados de oro. La noticia de la existencia de un modelo de arcilla es muy interesante porque son muy pocos los datos que tenemos sobre la escultura en Valencia en esta primera mitad del siglo XVI, la mayoría de las obras han desaparecido y los procesos de creación de las mismas apenas han quedado registrados. También tenemos que reseñar que estos dos nombres, Baltasar Fortuny ${ }^{28}$ y Martín Sancho, no son excesivamente renombrados como artistas en esta época. Solamente sabemos que Martín Sancho es un imaginero documentado como hijo del carpintero Vicente Sancho y hermano de un maestro homónimo también carpintero, pero desconocemos datos de obras ${ }^{29}$.

El retablo aparece claramente descrito en la capitulación. Aún mantenía una forma tradicional con zona central, escenas laterales, espiga, guardapolvos y banco. Se trataba de una obra que incidía en los temas relacionados con la cofradía y que fundamentalmente recogía los Siete derramamientos de sangre de Cristo en sus respectivas escenas, rodeando una zona central donde se situaba la figura del Ecce Homo. Estas escenas serían la circuncisión, el sudor de gotas de sangre en el huerto de los olivos, la flagelación, la coronación de espinas, la crucifixión con el derramamiento de sangre en manos y otra escena con los clavos en los pies y Jesús con la lanza en el costado. Completaba este conjunto una octava escena con el tema de los diez mil mártires. En la parte superior, la espiga estaba formada por un Dios Padre flanqueado por el mal y el buen ladrón. En los guardapolvos figuras de profetas que profetizaron la Pasión de Cristo. Una parte interesante era la zona inferior situada en el banco del retablo donde se debía representar la procesión que los disciplinantes de la cofradía realizaban el Jueves Santo.

Aunque se ha señalado como acicate para la extensión de la devoción a la Sangre de Cristo la publicación en Valencia del texto del notario Pineda que iba acompañado de unos grabados, éstos en realidad eran muy rudimentarios y, por otro lado, tratan unos temas que ya eran muy conocidos en la pintura valenciana anterior. Debieron servir para concretar exactamente las siete escenas que no se explicitan en el contrato pues se da por supuesto que se saben identificar, más que como modelos en sí mismos para la composición de las mismas. La iconografía general del retablo por tanto se enmarca en todo un proceso que ya había comenzado en la ciudad de Valencia con un cambio en la mentalidad por las devociones auspiciadas a partir de movimientos como la Estricta Observancia y el culto a sus grandes figuras, Bernardino de Siena y Catalina de Siena. Entre ellas también se generalizó el culto a la Sangre de Cristo favorecido por esta santa a la que en determinadas tablas se asocia directamente recogiendo la sangre del costado de Cristo ${ }^{30}$. Desde fines del siglo XV, la pintura valenciana está llena de imágenes de Cristo azotado en la columna o Cristo como Varón de dolores, que se vienen a sumar a las más frecuentes de Cristo crucificado. De hecho son varios los precedentes en la pintura de Vicente Macip que tratan este tema, como el Tríptico procedente de los Servitas de Sagunto, con una tabla central del Cristo

\footnotetext{
28 De Baltasar Fortuny no hemos localizado datos, quizá emparentado con un carpintero de nombre Pere Fortuny de quien se conocen obras para particulares desde 1495, y que en 1512 trabajaba en el Hospital General de Valencia, ver Gómez-Ferrer, 1997: 324.

${ }^{29}$ APPV, notario: Dionis Climent, 10775, 11 de marzo de 1541, arriendan ambos hermanos una casa. Por otro lado, sabemos que Vicent Sancho se examina del oficio de carpintero el 4 de septiembre de 1540, realizando "un bastiment per pintar a la romana ab son fris, cornisa y alquitrau", ARV, Gremio de carpinteros, libro 181.

${ }^{30}$ Sobre estos cambios iconográficos ver, Falomir, 1996: 344-350.
} 


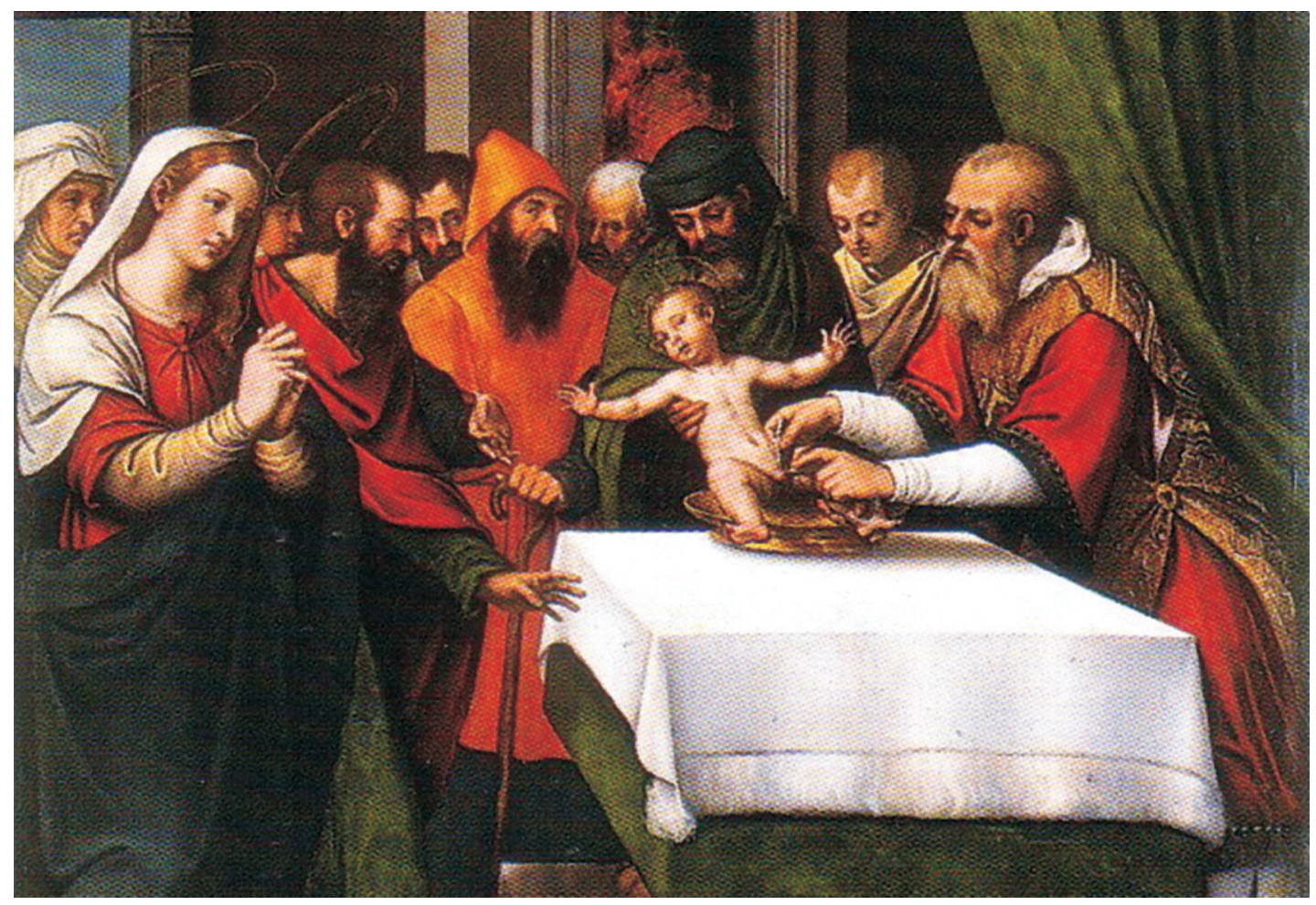

Fig. 3. Escena de la circuncisión del retablo del Gremio de Pelaires de la iglesia de San Nicolás de Valencia de Juan de Juanes.

patiens, lacerado con las sangre manando de sus manos y un cáliz en el que se recoge la sangre de la herida del costado. A los lados, la flagelación y el descendimiento. O los varios Ecce Homos coronados por espinas que se atribuyen a Vicente Macip (colección particular Paris, Barcelona, Museo Diocesano). Por otro lado, algunas de las escenas reseñadas de los siete derramamientos de la sangre de Cristo son también abundantes en el conjunto posterior de la obra de Juanes, la flagelación, oración en el Huerto, coronación de espinas o crucifixión se perfilan como escenas de predelas o escenas principales en algunos retablos. Incluso la procesión de los mil mártires y la circuncisión, con el explícito derramamiento de sangre sobre un plato, también forman parte de su quehacer pictórico y están presentes en el retablo del Gremio de Pelaires, conservado en la iglesia de San Nicolás de Valencia.

Sin embargo, hay dos escenas que resultan más complejas y desde luego mucho menos habituales, por un lado la espiga y por otro la predela, independientemente de los guardapolvos donde se debían pintar profetas, quizá Isaías o Moisés ${ }^{31}$. La espiga se presenta como una coronación del retablo en la que tiene que figurar un Dios Padre, hasta aquí nada sorprendente, pues era un tema que ya había sido empleado por Juanes en el retablo del gremio de plateros y en otras tablas como el Bautismo de Cristo. Pero en este caso se acompañaba por el buen y mal ladrón a cada lado, una representación que no es habitual en los retablos del obrador.

31 Moisés aunque no se pueda considerar estrictamente un profeta antecede a Cristo en el derramamiento de sangre para liberar al pueblo de Egipto. 
También la iconografía de la predela es difícil de precisar porque no se ha conservado ninguna representación de este tipo de procesiones de disciplinantes fechada en el siglo XVI. Sabemos que la de Valencia incluía cofrades como flagelantes, pues luego tenían que curar sus heridas al terminar la procesión en la casa de la cofradía. De hecho, en diciembre de 1543 la cofradía arrienda el hostal de la carretería contiguo a sus casas con la condición de que la puerta medianera entre el hostal y la cofradía estuviera abierta todos los domingos y fiestas y, especialmente, la noche de jueves santo, para que el arrendador diera entrada y salida a los disciplinantes y les permitiera luego tener un sitio con una caldera donde cocer el vino para sus curas ${ }^{32}$. Una de las pocas representaciones de este tipo de procesión en Valencia, aunque ya realizada en una etapa muy posterior, pertenece a Francisco de Goya, titulada precisamente Procesión de Valencia, con un grupo que acompaña una talla de Cristo crucificado, fechada hacia 1812, se conserva en la Fundación Bührle de Zurich ${ }^{33}$. Y en este sentido podemos imaginar que quizá la talla del Ecce Homo se sacara también en la procesión de los disciplinantes. Una escena real de la vida de Valencia en esa fecha es un tema excepcional en la producción pictórica del siglo XVI, y no encontramos ninguna otra representación de procesiones o hechos históricos concretos casi hasta la representación de la procesión de la llegada de la reliquia de San Vicente que aparece en la iglesia del Patriarca de Valencia de la mano de Bartolomé Matarana. No obstante, se puede encontrar un precedente de una narración con visos de realidad en la predela del retablo de San Vicente que estuvo en la iglesia de la Sangre de Segorbe atribuida al taller de los Macip, donde se representan dos escenas de la vida de San Vicente, la milagrosa cura de enfermos y la curación de apestados a la muerte de San Vicente, escenas todas ellas con composiciones muy vivas que intentan traducir unos hechos de forma verosímil.

\section{El retablo de la Sangre en el marco de la producción pictórica de los Macip}

El retablo de la Cofradía de la Sangre se sitúa por tanto en esa franja cronológica objeto de una cierta controversia, en que coinciden trabajando Vicente y su hijo Juan Macip, aunque los términos del mismo vienen a incidir en lo que la crítica historiográfica con mucho acierto está asumiendo en los últimos años. Por un lado, se advierte que el contrato se formaliza con ambos pintores, con unas cláusulas prácticamente coincidentes con las que años antes habían suscrito con otro colectivo, el gremio de plateros que les había encargado un retablo sobre el tema de San Eloy en $1534^{34}$. Este retablo dels argenters debía estar terminado en un plazo de cinco años y debieron cumplir con lo estipulado porque en 1539 estaban en condiciones de asumir otro gran encargo. Entre tanto, habían aceptado algunas otras obras de carácter menor como la terminación de unas pinturas para el retablo de San Bartolomé contratadas también por ambos pintores en 1537.

Vicente Macip sería la cabeza del taller, aunque desde 1534 se esté dando especial importancia a la presencia de su hijo en la ejecución de los retablos, presencia constatada de forma clara

32 APPV, 16052, 23 de diciembre de 1543, concesión a Francisco Badenes hostalero de los "hostal de la carreteria sive la navada ques deya mes chiqua del Spital de la Reyna e huy de la confraria y lo corral y les cambres que ixen al parador ab pacte y condicio que la porta migera que està entre la esglesia de la dita loable confraria e lo dit hostal aja de estar e estiga uberta tots los diumenges e festes y dies acostumats ab pacte y condicio que la nit de dijous sant de la semana santa los dits arrendador hajen de donar entrada y eixida al ramat dels dits disciplinants de dita confraria per la sua navada ço es per lo dit hostal y corral y per la porta del carrero al costat del monestir". Le piden tener un lugar para la caldera donde cuecen el vino los días de Jueves Santo para curar a los disciplinantes.

${ }^{33}$ Rodríguez G. de Ceballos, 2008: 389-405.

34 Sobre el retablo de San Eloy ver Benito, y Vallés, 1991: 353-361. 
en los documentos que exigen su intervención. Prácticamente hasta ese año todos los contratos se formalizaban con Vicente Macip y así había sucedido con el gran retablo de la catedral de Segorbe, aunque el nombre de Juan ya aparece en la documentación de este retablo cobrando estrenas en 1531. De nuevo, por otro documento inédito, sabemos que el 10 de septiembre de 1533 termina la formación de un pintor castellano oriundo de Motilla (¿Motilla del Palancar?) de nombre Juan Peligero, hijo de un ganadero y labrador del mismo nombre, que había permanecido durante seis años en su taller y también se menciona a Vicente Macip como el pintor que lo ha formado ${ }^{35}$. Pero este dato nos viene a confirmar la dificultad muchas veces de aceptar como obras únicas de un pintor los retablos realizados por un gran taller y quizá explica también las desigualdades existentes en algunas de estas obras. No acabamos tampoco de identificar a este pintor, quizá regresó a Castilla o se trasladó a Aragón, donde en fechas posteriores se documentan retablistas homónimos ${ }^{36}$.

Igual que en el citado retablo del gremi de argenters, el retablo de la Cofradía de la Sangre se suscribe con Vicente Macip y con Joan Macip, pare e fill, pero hay una claúsula específica que se encuentra en ambos contratos y que viene a incidir sobre la destacada personalidad del hijo en estas fechas, "que tota la desus dita pintura del dit retaule haja de eser y sia feta per lo dit en Joan Macip pintor fill del dit mestre Vicent Macip lo qual haja de pintar y acabar molt be e ab tota perfectio lo dit retaule segons que aquell millor pora e sabra pintar y daurar aquell". Aunque el taller seguía en manos de su padre, el verdaderamente afamado era Juanes, y es por tanto la segunda vez que en un contrato se especifica claramente el deseo de los comitentes de que la pintura sea obra del pintor más joven. En el caso del encargo de la iglesia de San Bartolomé de 1537 esto no había sucedido porque se trataba tan solo de rehacer el guardapolvos y pintar una espiga y no de un retablo completo, y se había capitulado con los dos, Vicente y Juan Macip, sin especificar quién debía pintar. Pero en 1539, no nos debe extrañar; la pericia de Juanes que aún era velada en el retablo de Segorbe, había quedado claramente demostrada en el retablo de San Eloy donde también se exigió expresamente que la pintura fuera de su mano, dejando a su padre en las labores de dorado ${ }^{37}$. Los miembros de la Cofradía de la Sangre conocían perfectamente este retablo, que para entonces ya debía estar concluido y expuesto en la capilla que tenían en la iglesia de Santa Catalina. Además se da la coincidencia de que en la firma de ambos contratos participa una misma personalidad, el platero Pere Mir, que fue mayoral del gremio de plateros cuando encargan la obra a los Macip en 1534 y que en 1539 era el clavario principal de la Cofradía de la Sangre y es uno de los que encarga el retablo.

Otros elementos de la capitulación también son bastante similares, el tiempo para su ejecución, el modo de pago, etc., teniendo en cuenta que ambos contratos se plantean ante el mismo notario y con pocos años de diferencia. Por el presente retablo, que los Macip debían hacer en un plazo de seis años, cobrarían ciento cincuenta ducados de oro en seis pagas, a medida que fueran entregando las piezas, pagadas en el día de Pascua de Resurrección.

La noticia del contrato de este retablo llena un gran vacío documental existente en el conjunto de la obra de Juanes. Tras haber entregado el retablo de los plateros encargado en 1534 y que debía estar concluido en 5 años, por tanto hacia 1539, no había ningún gran retablo documentado hasta el contrato suscrito el 29 de diciembre de 1548 con Juan de Juanes para Fuente la Hi-

\footnotetext{
35 APPV; notario: Francesc Vilar, 16607, 10 de septiembre de 1533, “Joan Pellejero pintor fill de Joan Pellejero quondam requero e laurador de la vila de la Mutilla del regne de Castella gratis fiat apoca a mestre Vicent Macip pintor present que he rebut les robes noves y lo vestit per raon dels sis anys quel ha servit en aprendre lo ofici de pintor en sa casa Et quia hec Actum Valencie, Testes Jacme Esteve mercader et Juan Orient torcedor de seda".

${ }^{36}$ Un Juan Peligero escultor firma unas capitulaciones para la realización de un retablo de la Soledad en la iglesia de Santa María de Daroca en 1675, Arce Oliva, 2003: 206-207.

${ }^{37}$ El alcance de esta cláusula fue puesto de relieve por Company, y Framis, 1999a: 263-278.
} 
guera $^{38}$. Coincide en cierta medida en un periodo en el que aunque Vicente Macip aún vivía no debía encontrarse con excesiva salud, dada su avanzada edad y al hecho de que en 1545 ya hubiera redactado testamento, aunque no fallece hasta 1551. La ejecución del retablo, si se produjo en el plazo de seis años como estaba estipulado, debió ocupar a Juanes durante los años 1540 a 1546. Con lo que luego estaría en condiciones de encargar el de Fuente la Higuera, quizá entonces contando ya con la colaboración del pintor Gaspar Requena que también se encontraba en la citada población y con quien se le asocia desde $1550^{39}$.

Con posterioridad a la firma del contrato no encontramos noticia alguna del retablo. No aparece mencionado en ningún texto que trata sobre la obra de Juanes, ni en aquellos iniciales como el del canónigo Vitoria ${ }^{40}$ donde se señalan algunas de sus obras en Valencia, ni en el texto de Orellana que también recopila algunos datos sobre las obras de Juanes. Tampoco aparece en las escasas descripciones que tenemos sobre la iglesia de la Cofradía de la Sangre. Las más completas son las que proporciona el Marqués de Cruilles en 1876, en las que sí que parece intuirse que al menos la imagen del Ecce Homo aún se conservaba. Se indica que era una imagen de Nuestro Señor colocada en el nicho principal del retablo, a donde fue trasladada de nuevo tras la reforma y nueva bendición de la iglesia de 1766. Quizá entonces solo conservaron la imagen, porque el retablo no está descrito y solo se menciona que constaba de dos cuerpos de orden compuesto y adornos platerescos, sin referencia alguna a pinturas ${ }^{41}$. No encontramos tampoco restos claros de este retablo como tal aunque su iconografía es frecuente en la obra de Juanes. Temas como la circuncisión o los santos mártires, como hemos señalado, se encuentran presentes en el retablo del gremio de pelaires, y no digamos la representación de escenas de oración en el Huerto, flagelación o crucifixión que se repiten a lo largo de su producción pictórica. Pero, por el momento, no podemos identificarlo con ninguna obra concreta.

\section{Conclusión}

El hallazgo de estas capitulaciones refuerza las hipótesis que se vienen esgrimiendo en las últimas investigaciones que permiten concretar a Juan Macip como el pintor más importante del taller de los Macip durante la década de los 30, llena un vacío documental entre la entrega del retablo del gremio de plateros y la contratación del retablo de Fuente la Higuera, y permite constatar el afianzamiento de determinados temas de carácter religioso en función de devociones populares fuertemente arraigadas en la Valencia del siglo XVI, al tiempo que introduce nuevas iconografías en el retablo como el hecho de pedir que la tabla del banco representara la propia procesión de los disciplinantes tal y como se realizaba el Jueves Santo.

\section{Apéndice Documental}

Documento 1. Contrato para la realización de la pintura del retablo de la Cofradía de la Sangre de Cristo de Valencia con Vicente y Juan Macip

Archivo del Colegio del Patriarca, Valencia, Protocolos notariales (APPV), n. ${ }^{\circ} 16049$, notario: Pere Mir 1539, diciembre, 29, Valencia

8 Sobre este retablo ver Pérez Burches, 2008: 25-33.

39 Pérez Burches, 2008, comenta acertadamente la relación entre Gaspar Requena y Juan de Juanes, pues ambos se encuentran juntos en Fuente la Higuera en septiembre de 1550 junto a sus respectivas esposas. También proporciona un dato interesante que puede ayudar a establecer la biografía de Juanes y es su matrimonio con Jerónima Comes entre marzo y septiembre de 1550 .

${ }^{40}$ Bassegoda, 1995: 165-172.

41 Cruilles, 1876. 
Die XXVIIII decembris anno a nativitate domini MDXXXX (es 1539)

In Dei nomine amen etc. nos Petrus Mir argentari clavarius laudabilis confratrie preciosissimi sanguinis domini nostri Jhesus Christi, Damianus Garcia panni parator, Laurentius del Mestre, sutor et Augustinus Aguilar eiusdem officii pani paratoris majorales in anno presenti dicte laudabilis confratrie dicto nominibus totam predictam laudabilem confratriam representantes presentis civitatis Valencie habitatores ex una et Vicentius Macip et Joannes Macip pictores pater et filius dicte civitatis habitatores gratis etc. cum presenti publico instrumento etc. confitemur nos partes predicte hoc est una pars nostrum alteri et altera alteri ad invicem et vicissim presentibus et acceptantibus quod in et super infrascriptis inter nos partes predictas fuerunt atque sunt facta inhita conventa et pactata concordata stipulata et promissa confirmata capitula in nostri parcium predictarum presentia per notarium infrascriptum alta et intelligibile voce lecta publicata quequide capitula sunt serie et thenoris sequentis:

En nom de nostre senyor Deu Jesucrist e de la Verge Maria mare sua capitols fets e fermats pactats e concordats per y entre los honorables en Pere Mir argenter en Damià Garcia perayre en Lorens del mestre sabater e en Agosti Aguillar perayre clavari e majorals en lo present any de la dita loable confraria de la preciosissima sanch de nostre señor Deu Jesu Christ y en dits noms representants tota la dita loable confraria de una part e los honorables en Vicent Macip e Joan Macip fill de aquell pintors de part altra en e sobre lo retaule que los sobredits en Vicent Macip e Joan Macip pintors eo l'altre de aquells han de pintar y daurar lo qual retaule la dita loable confraria ha de tenir dins les cases olim dites lo spital de la reyna e huy dites la confraria de la preciossisima sanch de Jesu Christ construhida en la dita ciutat de Valencia prop lo monestir del benaventurat sent Francesc los quals capitols son del serie e thenor inmediate seguent

E primerament es estat pactat, avengut e concordat entre les dites parts que lo retaule de fusta que de present sta y se ha de acabar y asentar en les cases de la dita loable confraria olim dites l'ospital de la reyna e huys nomenen la confraria de la preciosissima sanch de nostre senyor Deu Jhesu Christ, los dits mestre Vicent Macip e Joan Macip pintors pare e fill hajen de pintar y daurar y ab tota perfectio acabar lo dit retaule axi les istories com les guarnicions, polseres y obres mortes questan en lo dit retaule segons qui davall mes llargament se dira

Item es stat pactat avengut e concordat entre les dites parts que los dits clavari e majorals que huy son e per temps seran de la dita loable confraria hajen de donar e pagar als dits mestre Vicent Macip e Joan Macip pintors pare e fill o al altre de aquells per lo pintar e daurar del dit retaule segons dessus es dit e daval mes llargament se dira cent y cinquanta ducats de or y de bon pes pagadors en lo modo temps e forma davall specificador

Item es stat pactat avengut e concordat entre les dites parts que lo daurar y pintura del dessus dit retaule haja de esser y sia molt bona y ben acabada segons les forces dels dits pare e fill y de laltre de aquells abastaran e millor poran

Item es stat pactat havengut e concordat entre les dites parts (roto) del modo que Pilet?? (Pilato??) lo mostra als jueus dient ecce Homo que ha destar dins la pastera que ha de star en mig del dit retaule per lo semblant aquell haja de esser acabat e perfectionat tambe e ab tanta perfectio com totes les altres coses del dit retaule segons que millors los dessus dits y l'altre de aquells poran e sabran fer ab tota perfectio

Item es stat pactat e concordat entre les dites parts que les histories que san de pintar en lo dessus dit retaule son y han de esser huyt histories ço es les set scampaments que nostre señor Deu Jesu Christ feu y scampa la sua preciossisima sanch y damunt la dita pastera den mig y haja de esser pintada la historia dels deu milis martirs les quals histories hajen de esser pintades y acabades ab tota sa perfectio

Item en stat pactat e concordat entre les dites parts que en lo banch de davall del dit retaule se haja de pintar y que sia pintada la professo dels disciplçinants ques fa en la dita loable confraria e confrares de aquella lo dit del Dijous sanct

Item es stat pactat, avengut e concordat entre les dites parts que los dits mestre Vicent Macip e Joan Macip pare e fill sien obligats axi com ab los presents capitols se obliguen en acabar de pintar e daurar tot lo dit retaule en lo modo e forma dessus dits e segons que davall se dira dins termini de sis anys primer vinents e de la festa y dia de pasqua de resurectio primer vinent del present any en avant continuament contadors y menys si menys poran. En axi que aquell cascun any sien tenguts y obligats de donar e lliurar a la dita loable confraria e confrares de aquella pintada y ab tota perfectio acabada certa part del dit retaule que sia y mostre esser la sisena part del dit retaule poch mes o menys 
Item es stat pactat, avengut e concordat entre les dites parts que les polseres del dit retaule segons los departiments que y haura se hajen de pintar en aquelles profetes dels profetes qui profetizaren la pasio santissima de nostre redemptor Jhesu Christ excepto que dalt en la spiga en la una part de la polsera sia pintat lo sant lladre y en l'altra part de la polsera sia pintat lo mal lladre y en lo pla de la dita polsera y haja de esser pintat Deu lo pare

Item es stat pactat, avengut e concordat entre les dites parts que tota la dessus dita pintura del dit retaule haja de esser y sia feta per lo dit en Joan Macip pintor fill del dit mestre Vicent Macip lo qual haja de pintar y acabar molt be e ab tota perfectio lo dit retaule segons que aquell millor pora e sabra pintar y daurar aquell

Item es stat pactat, avengut e concordat entre les dites parts que los dits clavari e majorals de la dita loable confraria que huy son e per temps seran sien tenguts y obligats axi com ab los presents capitols se obliguen en donar e pagar als dits mestre Vicent e Joan Macip pare e fill pintors desus dits o a l'altre de aquells per lo pintar, daurar y acabar lo dessus dit retaule ab tota sa perfectio segons dessus es dit los dits cent cinquanta ducats de or e de bon pes, pagadors en esta forma ço es cascun any en lo dit dia y festa de pasqua de resurectio vint y cinch ducats escomensant la primera paga en la festa de pasqua de resurectio primer vinent del present any (de) mil cinchcents y quaranta e de aquí (en) avant cascun any vint y cinch ducats en dit termini e festa fins tant entregament sien pagats los dits cent cinquanta ducats de or y bon pes segons dessus es dit

Item es stat pactat, avengut e concordat entre les dites parts que dels presents capitols e de cascu de aquells sien fetes una e moltes cartes publiques e tantes quantes les dites parts ne volran executar ab submissio de for e variacio de juhi e clausula de no littigar e obligacio de bens juxta stil e practica del notari rebedor dels presents capitols

Quibus quidem capitulis lectis...

Testes huius rei sunt honorabiles Melchor Sanchiz mercator et Michael Sentis scriptor supradicte civitatis Valencie habitatores

Documento 2. Contrato para la realización de la estructura de madera del retablo con Baltasar Fortuny escultor

1539, noviembre, 17 , Valencia

APPV, 16048, notario: Pere Mir

Die XVII novembris anno a nativitate Domini MDXXXVIIII.

Ego Baltasar Fortuny sculptor presentis civitatis Valencie habitator. Gratis etc. cum presenti publico instrumento etc. promitto et fide bona convenio vobis honorabilibus clavaris et majoralibus laudabilis confratrie preciosissimi sanginus domini nostri Jhesu Christi presentis civitatis, absentibus etc. notarii quod infrascripto etc. stipulante etc. et vostris dictis nominibus presente tamen et ac reptante honorabile Petro Mir argentario clavario in anno presenti dicte laudabilis confratrie intra mes tres primo venturas et a die presenti in posterum continuo computandos dare et cum omni effectu tradere in domibus dicte laudabilis confratrie olim dictis lo Spital de la reyna retabulum seu tabulam nondum pictam per me facturum ab dicta confratriam quod quidem retabulum seu tabula isse debet viginti et quatuor palmarum de peu dret et quatordecim palmarum de latitudine totius luminis seu de tota llum cum suis polseriis et diffinicionibus sive ab ses polseres y deffinicions. Que quidem tabula debet esse conformis sive mostram de pictam seu factam in quodam papiro recondito et posito penes vos prefatum clavarium in medio cuius quodam retabuli seu tabule nondum picte y ha de haver una pastera in qua ponenda figura domini nostri Jhesu Crist sint Pilatus ipsum hostendit judeis dicens ecce homo. Que quidem tabula nondum picta debet esse et est facienda de fusta pini quam quidem fustam pini. Vos predicti clavarius et majorales predicte confratrie jam michi tradi dictis et dedistis et pro laboribus seu per les mans seu pro precio dicti retabuli solvere et paccare teneamini michi et meis quindecim ducatos auri justi et resti ponderis. Quos michi solvere et paccare teneamini facto dicto retabulo et ipso posito in suo loco intus dictam domum dicte confratrie preciosissimi sanguinis domini nostri Jhesu Crist. Et quo quidem retabulum in dicto termino sine tempore vobis dictis nominibus tradere promitto sub pena quinquaginta libraris monete regalium Valencie pro pena etc. pro quo quidem pena ffiat executoria large cum fori submissione variacione judicii et cum omnibus clausulis necessariis et oportunis et in similibus poni asuetis etc. et pro predictis 
omnibus et singulis etc. obligo vobis et vestris omnia et singula bona et jura mea mobilis etc. Quod est actum Valencie etc.

Testes huius rei sunt honorabilis Bernardinus Aguilar mercator et Michael Sentis, scriptor iam dicte civitatis Valencie habitatores.

Documento 3. Contrato para la realización de la figura del Ecce Homo del retablo con Martín Sancho, imaginero

1539, noviembre, 17, Valencia

APPV, 16048, notario: Pere Mir

Dictis die et anno (17/11/1539)

Ego Martinus Sancho ymaginarius sive ymaginari presentis civitatis Valencie habitatoris gratis etc. cum presenti publico instrumento etc. promitto et fide bona convenio vobis honorabilibus clavario et majoralibus laudabilis confratrie preciosissimi sanguinis domini nostri Jhesu Crist presentis civitatis absentibus etc. notarii quod infrascripto etc. stipulante etc. Et vostris dictis nominibus presente tamen et acceptante honorabile Petro Mir argentario clavario in anno presenti dicte laudabilis confratrie quod luit ad festum pasche resurectionis primo venturum anni quod presentis et subscripti vobis seu dicte laudabili confratrie cum omni effectu dabo hun ecce homo de bulto molt bo e ben acabat segons una mostra de argila que stá en poder del dit clavari de altaria de set palms y ab sa diadema y ab tots sos compliments de fusta de ciprer pro eius precio seu pro laboribus ipsius vos prefati clavarius et majorales teneamini michi solvere et paccare decem ducatos auri justi et recti ponderis. Que quidem predicta vestra et singulo complere et ad implere promitto et tenemi? sub pena viginti librarum pro pena etc. ratto pacto etc. pro quibus ffiat executoria large enmisori submissione variacioni judicii demostracione appellacionis juste frivole et injuste. Et clausulis juratis mon littigandi nech impretrandi sub pena quinquaginta solidos dicte monete regalium Valencie ultra perjuri penam etc. Et propie dictis omnibus etc. obligo vobis et vostris dictis nominibus omnis et singula bona et jura mea mobilia etc. Quod est actum Valencie etc.

Testes huius rei sunt honorabiles Michael Sentis scriptor et Bernardinus Aguila mercator predicte civitatis Valencie habitatores.

\section{BIBLIOGRAFÍA}

Albi, José, Joan de Joanes y su círculo artístico, Valencia, 1979.

Alejos, Asunción, "Cofradía de la Sangre", Valencia, Monumentos desaparecidos, Valencia, 1999, pp. 84-87.

Arce Oliva, Ernesto, "Retablos de los siglos XVI y XVII", Comarca del Campo de Daroca, Gobierno de Aragón, 2003, pp. 206-207.

Bassegoda, B., "Vicente Vitoria (1650-1709) primer historiador de Joan de Joanes", Locus Amoenus, 1995, pp. $165-172$.

Benito, Fernando y Vallés, Vicente, "Nuevas noticias de Vicente Macip y Joan de Joanes", Archivo Español de Arte, n. ${ }^{\circ} 255,1991$, pp. 353-361.

Benito, Fernando, "El Maestro de Cabanyes y Vicente Macip. Un solo artista en distintas etapas de su carrera", Archivo Español de Arte, Madrid, n. ${ }^{\circ}$ 263, 1993, pp. 223-244.

Benito, Fernando, Vicente Macip (1475-1550), Valencia, 1997.

Benito, Fernando, Joan de Joanes, Cat. Exp., Valencia, 2000.

Company, Ximo y Tolosa, Luisa, "De pintura valenciana. Bartolomé Bermejo, Rodrigo de Osona, El Maestro de Artés, Vicente Macip y Juan de Juanes", Archivo Español de Arte, 1999, pp. 263-278.

Company, Ximo y Tolosa, Luisa, "La obra de Vicente Macip que debe restituirse a Joan de Joanes", Archivo de Arte Valenciano, 1999, pp. 50-61.

Corbín, Juan Luis, La Plaza del Ayuntamiento, antigua de San Francisco, Caja de Ahorros de Valencia, 1988.

Cruilles, Marqués de, Guía Urbana de Valencia, Valencia, 1876, T. I., pp. 436-439.

Hernández, Lorenzo y Company, Ximo, "De nuevo sobre Joan Macip, alias Joan de Joanes", en De pintura Valenciana (1400-1600), Alicante, 2006, pp. 211-269.

Falomir, Miguel, Arte en Valencia, 1472-1522, Valencia, 1996, pp. 344-350.

Arch. esp. arte, LXXXv, 337, ENERO-MARZO 2012, 1-16, ISSN: 0004-0428 
Falomir, Miguel, "La construcción de un mito. Fortuna crítica de Juanes en los siglos XVI y XVII", Espacio, Tiempo y Forma, Serie VII, 12, 1999, pp. 123-147.

Falomir, Miguel, “Joanes y su entorno: relaciones sociales y afinidades culturales", en De pintura Valenciana (1400-1600), Alicante, 2006, pp. 271-288.

Gómez-Ferrer, Mercedes, Arquitectura en la Valencia del siglo XVI. El Hospital General y sus artífices, Valencia, 1997.

Labarga García, Fermín, "La devoción a las Cinco Llagas y a la Sangre de Cristo en las cofradías riojanas de la Vera Cruz", Zainak-Cuadernos de Antropologia-Etnografia, n. ${ }^{\circ}$ 18, Eusko Ikaskuntza, 1999, pp. 381392.

Martin Pineda, Andreu, Contemplació en honor y reverencia de les set vegades quel nostre redemptor Jesus escampa la sua preciosissima sanch ab les propietats de cascuna feta, Valencia, 1521.

Martínez Romero, Tomás, "L’obra profana d'Andreu Martí Pineda i la literatura valenciana a la primera meitat del XVI", Revista de la Societat Catalana de Llengua i Literatura, n. ${ }^{\circ}$ 12, Barcelona, 2001.

Navarro Espinach, Germán, "Las Cofradías de la Vera Cruz y de la Sangre de Cristo en la corona de Aragón (Siglos XIV-XVI)", Anuario de estudios medievales, Zaragoza, 2006, vol. 36, n. ${ }^{\circ}$ 2, pp. 583-611.

Pérez Burches, Inmaculada, "Aportación documental al retablo mayor de la iglesia de la Natividad de la Font de la Figuera de Joan de Joanes", Ars Longa, n. ${ }^{\circ}$ 17, 2008, pp. 25-33.

Rodríguez G. de Ceballos, Alfonso, "La procesión de Disciplinantes de la Academia de Bellas Artes de San Fernando. Goya y la religiosidad popular", Anales de Historia del Arte, Volumen Extraordinario, 2008, pp. 389-405.

Samper, Vicente, "Una tabla de Santa Ana, la Virgen y el Niño junto a María Magdalena, identificable con la central del retablo de los Joan (1507) de Vicente Macip", Archivo de Arte Valenciano, 1994, pp. 3538.

Samper, Vicente, "Documentos inéditos para la biografía de los Macip", Archivo Español de Arte, n. ${ }^{\circ} 294$, 2001, pp. 163-171.

Teixidor, Josef, Antigüedades de Valencia, El Archivo Valentino, Valencia, 1895.

Fecha de recepción: 3-III-2010

Fecha de aceptación: 9-XII-2010 